\title{
Sodium Borohydride Reduction of Aqueous Silver-Iron-Nickel Solutions: a Chemical Route to Synthesis of Low Thermal Expansion-High Conductivity Ag-Invar Alloys
}

\author{
E.A. STERLING, J. STOLK, L. HAFFORD, and M. GROSS
}

Thermal management is a critical concern in the design and performance of electronics systems. If heat extraction and thermal expansion are not properly addressed, the thermal mismatch among dissimilar materials may give rise to high thermal stresses or interfacial shear strains, and ultimately to premature system failure. In this article, we present a chemical synthesis process that yields Ag-Invar (64Fe-36Ni) alloys with a range of attractive properties for thermal management applications. Sodium borohydride reduction of an aqueous Ag-Fe-Ni metal salt solution produces nanocrystalline powders, and conventional powder processing converts this powder to fine-grained alloys. The samples are characterized by X-ray diffraction (XRD), scanning electron microscopy, thermomechanical analysis, and electrical conductivity measurements; thermal conductivity is estimated using the Wiedemann-Franz law. Sintering of $\mathrm{Ag}-\mathrm{Fe}-\mathrm{Ni}$ powders leads to the formation of two-phase silver-Invar alloys with low coefficients of thermal expansion (CTEs) and relatively high electrical conductivities. A sample of 50Ag-50Invar exhibits a CTE of $8.76 \mu \mathrm{m} /\left(\mathrm{m} \cdot{ }^{\circ} \mathrm{C}\right)$ and an estimated thermal conductivity of $236 \mathrm{~W} /(\mathrm{m} \cdot \mathrm{K})$. The Ag-Invar alloys offer thermodynamic stability and tailorable properties, and they may help address the need for improved packaging materials.

DOI: $10.1007 / \mathrm{s} 11661-009-9859-4$

(C) The Minerals, Metals \& Materials Society and ASM International 2009

\section{INTRODUCTION}

THERMAL management in electronics components, assemblies, and systems is a critical consideration as circuit board component density continues to rise and as new build-up processes and component integration advance. A major thermal issue facing high-performance circuit designers is the control of thermal energy that integrated circuits (ICs) generate during operation. Effective removal of heat necessitates the use of materials with physical properties dissimilar to those of the semiconductor devices.

Heat generation and coefficient of thermal expansion (CTE) mismatch among semiconductor devices, interconnects, and packaging materials are known to affect electronic assembly performance and reliability. Semiconductors and ceramic packaging materials naturally exhibit low thermal conductivity and low thermal expansion, while the highly conductive alloys that are effective in electrically connecting components and removing heat typically exhibit relatively large CTEs. Further, composite package substrates and underfill materials tend to exhibit low thermal conductivity but relatively high CTEs. If heat extraction is not addressed,

E.A. STERLING and L. HAFFORD, Students, and J. STOLK, Associate Professor, are with the Department of Mechanical Engineering and Materials Science, Franklin W. Olin College of Engineering, Needham, MA 02492. Contact e-mail: stolk@olin.edu M. GROSS, Assistant Professor, is with the Department of Chemical Engineering, Bucknell University, Lewisburg, PA 17837.

Manuscript submitted November 25, 2008.

Article published online June 5, 2009 the thermally sensitive devices may cease to function. If differential thermal expansion is not addressed, thermal mismatch among dissimilar materials may give rise to high thermal stresses or interfacial shear strains, and ultimately lead to crack formation and premature component failure.

\section{A. Substrate-Based Thermal Management}

The device or IC substrate represents a key opportunity for improvement of thermal management, and much attention in recent years has centered on the design of substrates that reduce thermal strain, aid heat dissipation, or both. Two approaches employed by circuit designers for substrate-based thermal management include laminate substrates with core constraining layers and substrates with heat sinks or heat spreaders directly joined to devices. Both of these approaches effectively reduce heat or thermal strain, and thereby increase the reliability of modern microelectronic or optoelectronic systems. Successful implementations of low CTE, high conductivity core constraining layers include clad metals such as copper-Invar-copper, ${ }^{[1-3]}$ carbon fiber-based cores, ${ }^{[4,5]}$ and carbon-SiC laminate composites ${ }^{[6]}$ A number of composite packaging materials have been explored for use as thermal management substrates in thermally demanding components such as power amplifiers, laser diodes, thermoelectric coolers, and radio frequency (RF) and microwave devices. These include metal matrix composites, ${ }^{[7-11]}$ metal-metal composites, ${ }^{[8,9,12-14]}$ and advanced diamond or graphite films and composites. ${ }^{[7]}$ 


\section{B. Ag-Fe-Ni for Thermal Management}

Although many of the composites and advanced alloys used in core constraining and heat sink or heat spreading applications have demonstrated desirable thermal expansion and conductivity properties, some also exhibit significant anisotropy, high material or fabrication costs, high density, poor interfacial characteristics, toxicity, or difficult machinability or processing. This article presents an alternative synthesis approach for the preparation of a range of nanocomposite $\mathrm{Ag}-\mathrm{Fe}-\mathrm{Ni}$ powders, and it describes the properties and phase development of fine-grained, low thermal expansionhigh conductivity Ag-Invar alloys formed from the nanoscale powders. The fine-grained $\mathrm{Ag}-\mathrm{Fe}-\mathrm{Ni}$ powder metallurgy alloys described here may offer the desired thermal, electrical, and physical properties necessary for thermal management in high-performance circuits.

Composites and alloys prepared by chemical synthesis and processing of nanoscale powders offer some distinct advantages over bulk materials, including better mechanical properties, increased densification rates, and higher purity in the bulk alloys. In addition, the intimately mixed nanoscale powders produced by chemical synthesis exhibit phase evolution, diffusion, and interfacial energy properties and behaviors that more closely match those expected in thin films-scale metallic materials. The Ag-Fe-Ni alloys investigated in this study may also help researchers address manufacturing issues of increasing importance. The Ag-Fe-Ni alloys are ductile, and they may be easily formed into other shapes by punching, shearing, bending, drawing, and extruding. Disadvantages of Ag-Fe-Ni include high material costs and relatively high density compared to some of the metal matrix composites and carbon-based thermal management materials. In addition, the low Young's modulus of $\mathrm{Ag}-\mathrm{Fe}-\mathrm{Ni}$ alloys may be advantageous in applications involving direct mounting of devices to heat extracting substrates, but it could also serve as a concern for core constraining applications that rely on high laminate composite stiffness to reduce thermal strains in the electronic assembly.

Like many of the core constraining composites described previously, the alloys developed in this study leverage the thermal properties of Invar, a face-centeredcubic alloy of $64 \mathrm{wt}$ pct $\mathrm{Fe}$ and $36 \mathrm{wt}$ pct nickel. Invar and near-Invar alloys exhibit zero, negative, or very low thermal expansion near ambient temperatures as a result of a balance between thermal expansion and volume magnetostriction. ${ }^{[15]}$ Above room temperature, the CTE of Invar gradually increases, with more marked changes in CTE occurring above temperatures of approximately $225^{\circ} \mathrm{C}$, where the thermal expansion is no longer balanced by the magnetostriction.

This investigation of $\mathrm{Ag}-\mathrm{Fe}-\mathrm{Ni}$ alloys builds on prior work in powder metallurgy $\mathrm{Cu}$-Invar thermal management materials. Although copper appears to be a logical choice for the high-conductivity phase of an Invar-based composite, investigations of powder $\mathrm{Cu}-\mathrm{Fe}-\mathrm{Ni}$ alloys have demonstrated that production of metallurgically stable, two-phase, low thermal expansion-high conductivity alloys in this system is difficult. The solid-state solubility limits in the ternary $\mathrm{Cu}-\mathrm{Fe}-\mathrm{Ni}$ system present a significant challenge to powder processing of these materials, as diffusion of $\mathrm{Fe}$ and $\mathrm{Ni}$ into the $\mathrm{Cu}$ has deleterious effects on both phases. Iron and nickel impurities dramatically decrease the electrical and thermal conductivity of the $\mathrm{Cu}$ phase, ${ }^{[16]}$ and changes in the $\mathrm{Fe}-\mathrm{Ni}$ ratio increase the thermal expansion of the Invar phase. ${ }^{[17]}$

Silver offers a promising alternative to copper for use as a high conductivity phase. Although little is published on the phase development and ambient-temperature phase stability in the Ag-Fe-Ni ternary system, the $\mathrm{Ag}-\mathrm{Ni}, \mathrm{Ag}-\mathrm{Fe}$, and $\mathrm{Fe}-\mathrm{Ni}$ binary phase diagrams indicate that the formation of a range of thermodynamically stable, two-phase Ag-Invar materials using chemical synthesis and powder processing techniques is possible. The feasibility of $\mathrm{Ag}-\mathrm{Fe}-\mathrm{Ni}$ alloy use requires that the $\mathrm{Ag}$ and Invar phases remain metallurgically unmixed to avoid the harmful effects of impurities on $\mathrm{Ag}$ conductivity. Silver-Invar composites in a limited compositional range have been produced by Ag liquid infiltration of an Invar powder compact; ${ }^{[9,18]}$ and these alloys have been used in some GaAs IC thermal management applications. The phase stability, microstructural development, and properties of ternary Ag-Fe-Ni alloys produced from nanocrystalline metal powders, however, have not been thoroughly investigated.

The present study aims at the production of nanocrystalline $\mathrm{Ag}-\mathrm{Fe}-\mathrm{Ni}$ powders using solution-based chemical synthesis techniques; formation of fine-grained, low CTE-high conductivity Ag-Invar alloys by conventional powder processing and heat treatment; and characterization of the resulting products. This exploration of a wide compositional range of fine-grained $\mathrm{Ag}$ Invar alloys has the potential to provide insights into the behavior and performance of these promising ternary alloys and to reveal Ag-Invar compositions that are useful in a variety of thermal management applications.

\section{EXPERIMENTAL PROCEDURES}

\section{A. Chemical Synthesis}

Nanocrystalline Ag-Fe-Ni powders were prepared using silver nitrate $\left(\mathrm{AgNO}_{3}\right)$, ferric nitrate $\left(\mathrm{Fe}\left(\mathrm{NO}_{3}\right)_{3}\right.$. $\left.9 \mathrm{H}_{2} \mathrm{O}\right)$, and nickel nitrate $\left(\mathrm{Ni}\left(\mathrm{NO}_{3}\right)_{2} \cdot 6 \mathrm{H}_{2} \mathrm{O}\right)$ using a procedure previously reported by the author. ${ }^{[19]}$ The nominal iron to nickel ratio for all synthesis reactions was maintained at $1.78: 1$, a ratio corresponding to the low expansion Invar alloy. The metal salts were collectively diluted to a $0.15 \mathrm{M}$ aqueous solution. Sodium borohydride $\left(\mathrm{NaBH}_{4}\right)$ was added to the solution at ambient temperature under constant magnetic stirring. The molar ratio of $\mathrm{NaBH}_{4}$ to transition metal ions in the reaction mixture was 2:1 to ensure complete reduction of metal ions. The reaction resulted in a reduction of $\mathrm{Ag}^{+}$, $\mathrm{Fe}^{3+}$, and $\mathrm{Ni}^{2+}$ to metallic $\mathrm{Ag}, \mathrm{Fe}$, and $\mathrm{Ni}$ in the form of a fine precipitate. Other reaction products may include sodium hydroxide $(\mathrm{NaOH})$, ammonia $\left(\mathrm{NH}_{3}\right)$, boric acid $\left(\mathrm{B}(\mathrm{OH})_{3}\right)$, and possibly some metal borates. Precipitate color ranged from light brownish gray for 
the Ag powder, to black for samples of high Fe-Ni concentration. A possible overall reaction for the reduction of $\mathrm{Ag}$ is

$$
\begin{aligned}
& 2 \mathrm{AgNO}_{3}+4 \mathrm{NaBH}_{4}+10 \mathrm{H}_{2} \mathrm{O} \\
& \quad \rightarrow 2 \mathrm{Ag}+2 \mathrm{NH}_{3}+4 \mathrm{NaOH}+4 \mathrm{~B}(\mathrm{OH})_{3}+7 \mathrm{H}_{2}
\end{aligned}
$$

A possible overall reaction for the reduction of $\mathrm{Ni}$ is

$$
\begin{aligned}
& \mathrm{Ni}\left(\mathrm{NO}_{3}\right)_{2} \cdot 6 \mathrm{H}_{2} \mathrm{O}+4 \mathrm{NaBH}_{4}+4 \mathrm{H}_{2} \mathrm{O} \\
& \quad \rightarrow \mathrm{Ni}+2 \mathrm{NH}_{3}+4 \mathrm{NaOH}+4 \mathrm{~B}(\mathrm{OH})_{3}+7 \mathrm{H}_{2}
\end{aligned}
$$

A possible overall reaction for the reduction of $\mathrm{Fe}$ is

$$
\begin{aligned}
& 2 \mathrm{Fe}\left(\mathrm{NO}_{3}\right)_{3} \cdot 9 \mathrm{H}_{2} \mathrm{O}+10 \mathrm{NaBH}_{4}+4 \mathrm{H}_{2} \mathrm{O} \\
& \quad \rightarrow 2 \mathrm{Fe}+6 \mathrm{NH}_{3}+10 \mathrm{NaOH}+10 \mathrm{~B}(\mathrm{OH})_{3}+13 \mathrm{H}_{2}
\end{aligned}
$$

The resulting nanoscale powders were filtered using a fritted glass filtration funnel and vacuum assist; rinsed with warm $\left(\sim 90{ }^{\circ} \mathrm{C}\right)$ deionized water to assist in the removal of $\mathrm{NH}_{3}, \mathrm{NaOH}$, and $\mathrm{B}(\mathrm{OH})_{3}$; and dried with acetone. The powders were heat treated in a $\mathrm{H}_{2}$ atmosphere at $520{ }^{\circ} \mathrm{C}$ to reduce any oxides present in the sample and to dissociate any metal borates that remained after initial washing into $\mathrm{B}_{2} \mathrm{O}_{3}$ and metal particles. After heat treatment, the powders were washed with warm water to remove $\mathrm{B}_{2} \mathrm{O}_{3}$, filtered, and dried with acetone.

Heat-treated powders were compacted in stainless steel pellet dies. A 5 -mm pellet die with $0.3 \mathrm{~g}$ powder was used to produce small cylindrical pellets for thermal expansion measurements, and a $25-\mathrm{mm}$ pellet die with $3 \mathrm{~g}$ powder was used to create coin-shaped pellets for use in all other analyses. The pellets were sintered in a $\mathrm{H}_{2}$ atmosphere at $900{ }^{\circ} \mathrm{C}$ for 1 hour to yield fine-grained Ag-Invar alloys.

\section{B. Characterization Techniques}

The crystalline phases of the as-synthesized powders, heat-treated powders, and sintered pellets were characterized using a Shimadzu MAXima XRD-7000 X-ray powder diffractometer (Shimadzu Corporation, Kyoto, Japan). A copper $\mathrm{Cu} K_{\alpha}$ radiation source at $40 \mathrm{kV}$ and $30 \mathrm{~mA}$ was used for all X-ray diffraction (XRD) measurements, and XRD data processing included background subtraction, smoothing, and $\mathrm{Cu} K_{\alpha 2}$ reflection subtraction. Compositions of the sintered pellets were measured using a Shimadzu AA-6650 atomic absorption spectrophotometer (AAS, Shimadzu Corporation, Kyoto, Japan) in flame mode. For the AAS analyses, approximately $0.5 \mathrm{~g}$ of the solid samples was dissolved in nitric acid and serially diluted until the metal content of the dilution was nominally between 1 and 5 ppm.

Particle sizes of the as-prepared and heat-treated powders were characterized using a JEOL* 200CX

*JEOL is a trademark of Japan Electron Optics Ltd., Tokyo.

transmission electron microscope (TEM). All TEM samples were prepared by ultrasonically dispersing powders in acetone and dropping the dispersed powder onto holey carbon $\mathrm{Cu}$ grids. Sintered pellets were ground with $\mathrm{SiC}$ papers and polished to $0.05-\mu \mathrm{m} \mathrm{Al}_{2} \mathrm{O}_{3}$, and a JEOL 6060LV scanning electron microscope was used to characterize the microstructures of the sintered pellets. Phase compositions were investigated by compositional dot mapping using a Thermo Scientific Noran System SIX energy dispersive spectrometry (EDS) microanalysis system (Thermo Scientific, Waltham, MA).

The density of the sintered pellets was determined through manual volume measurements and analytical balance mass measurements. Thermal expansion was evaluated from $-20{ }^{\circ} \mathrm{C}$ to $320{ }^{\circ} \mathrm{C}$ with a TA Instruments TMA 2940 thermomechanical analyzer (TMA, TA Instruments, New Castle, DE) in accordance with the ASTM E831 standard test method for linear thermal expansion of solid materials. The thermal expansion $v s$ temperature data were used to calculate the average CTE, $\alpha$, over the temperature range of $20^{\circ} \mathrm{C}$ to $100{ }^{\circ} \mathrm{C}$ using Eq. [1]:

$$
\alpha=\frac{1 \Delta l}{l_{o} \Delta T}
$$

where $T$ is temperature in ${ }^{\circ} \mathrm{C}, l_{o}$ is the sample length at room temperature, and $l$ is the sample length at any given temperature.

The ambient temperature electrical conductivity of the large sintered samples was measured using a fourprobe technique of the Van Der Pauw configuration. ${ }^{[20]}$ A current of 2 A was applied by an Agilent E3632A DC power supply (Agilent Technologies, Inc., Santa Clara, CA), and voltage was measured by an Agilent 34,420-A nanovolt micro-Ohm meter. Electrical conductivity was calculated using Eq. [2]:

$$
\sigma=\frac{0.2207 \cdot I}{V \cdot t}
$$

where $\sigma$ is the electrical conductivity in $\mathrm{Ohm}^{-1} \cdot \mathrm{cm}^{-1}, I$ is the applied current in Amperes, $V$ is the voltage in volts, and $t$ is the thickness of the pellet in centimeters. Thermal conductivity values for all samples were estimated using the Wiedemann-Franz law, which relates thermal conductivity to electrical conductivity according to Eq. [3]:

$$
\kappa=\frac{\pi^{2}}{3}\left(\frac{\mathrm{k}}{e}\right)^{2} \sigma T
$$

where $\kappa$ is the thermal conductivity, $\sigma$ is the electrical conductivity, $T$ is the absolute temperature, $\mathrm{k}$ is the Boltzmann's constant, and $e$ is the electron charge. ${ }^{[21]}$ The Wiedemann-Franz law provides a good approximation of the thermal conductivity of materials in which electrons (as opposed to phonons) are primarily responsible for heat conduction, as is the case with the $\mathrm{Ag}-\mathrm{Fe}-\mathrm{Ni}$ metallic alloys considered in this study.

\section{RESULTS AND DISCUSSION}

\section{A. Sample Composition and Phase Identification}

Nominal compositions and actual compositions as determined by AAS of the heat-treated powders are 
Table I. Sample Identification, Nominal Compositions, and Actual Compositions as Determined by AAS and Fe:Ni Ratio in the Sintered Ag-Fe-Ni Pellets

\begin{tabular}{|c|c|c|c|c|c|c|c|}
\hline \multirow[b]{2}{*}{ Sample } & \multicolumn{3}{|c|}{ Nominal Composition, Wt Pct } & \multicolumn{3}{|c|}{ Actual Composition, Wt Pct } & \multirow[b]{2}{*}{ Fe:Ni Ratio } \\
\hline & $\mathrm{Ag}$ & $\mathrm{Fe}$ & $\mathrm{Ni}$ & $\mathrm{Ag}$ & $\mathrm{Fe}$ & $\mathrm{Ni}$ & \\
\hline 90Ag-10Invar & 90.00 & 6.40 & 3.60 & 89.04 & 7.29 & 3.67 & $1.99: 1$ \\
\hline 80Ag-20Invar & 80.00 & 12.80 & 7.20 & 77.83 & 14.90 & 7.27 & 2.05:1 \\
\hline 70Ag-30Invar & 70.00 & 19.20 & 10.80 & 67.14 & 21.32 & 11.54 & $1.85: 1$ \\
\hline 60Ag-40Invar & 60.00 & 25.60 & 14.40 & 56.48 & 28.02 & 15.50 & $1.81: 1$ \\
\hline $50 \mathrm{Ag}-50 \mathrm{Invar}$ & 50.00 & 32.00 & 18.00 & 48.97 & 32.74 & 18.28 & $1.79: 1$ \\
\hline 40Ag-60Invar & 40.00 & 38.40 & 21.60 & 38.99 & 38.92 & 22.10 & $1.76: 1$ \\
\hline 30Ag-70Invar & 30.00 & 44.80 & 25.20 & 30.65 & 44.04 & 25.32 & $1.74: 1$ \\
\hline 20Ag-80Invar & 20.00 & 51.20 & 28.80 & 22.53 & 49.08 & 28.38 & $1.73: 1$ \\
\hline 10Ag-90Invar & 10.00 & 57.60 & 32.40 & 10.38 & 56.92 & 32.70 & $1.74: 1$ \\
\hline
\end{tabular}

shown in Table I. The close agreement between the nominal and actual compositions indicates that $\mathrm{Ag}, \mathrm{Fe}$, and $\mathrm{Ni}$ are fully reduced by $\mathrm{NaBH}_{4}$ during synthesis. Table I also provides the Fe:Ni ratio in each Ag-Fe-Ni sample. Maintaining a Fe:Ni ratio close to that of Invar (1.78:1) is crucial to ensuring that the $\gamma-(\mathrm{Fe}, \mathrm{Ni})$ phase in the Ag-Fe-Ni alloys has low thermal expansion. Increasing iron content raises the CTE of Invar alloys faster than increasing nickel content, though shifting the Invar composition in either direction is detrimental to maintaining low CTE values.

The XRD patterns of the $30 \mathrm{Ag}$-70 Invar as-prepared powder, heat-treated powder, and sintered pellet are shown in Figure 1. The X-ray pattern of the as-prepared nanoscale powder has broad reflections from the metallic Ag phase and an oxide phase that corresponds to $\mathrm{NiFe}_{2} \mathrm{O}_{3}$ or $\mathrm{Fe}_{3} \mathrm{O}_{4}$. The low intensity reflection at approximately $43.5 \mathrm{deg}$ may correspond to one of these oxides, or possibly to metallic $\mathrm{Fe}, \mathrm{Ni}$, or $\gamma$-(Fe,Ni). Since the reflections are broad and the peaks from the various phases overlap, it is difficult to establish unambiguously whether the $\gamma$-(Fe,Ni) Invar phase is formed in the as-prepared samples. After the $520{ }^{\circ} \mathrm{C}$ heat treatment, reflections corresponding to metallic $\mathrm{Ag}$ and the facecentered-cubic $\gamma$-(Fe,Ni) Invar phase are clearly visible, indicating that the desired phases are formed in the processed Ag-Fe-Ni alloys. The Ag and Invar peaks become clearly defined after sintering at $900{ }^{\circ} \mathrm{C}$ due to grain growth in the Ag-Fe-Ni pellets.

The TEM micrographs of the as-prepared 50 Ag-50 Invar sample are shown in Figures 2 and 3. The asprepared powder has a bimodal distribution of grain sizes corresponding to the Ag phase (larger) and the $(\mathrm{Fe}, \mathrm{Ni})$ phase (smaller). The smaller grains, which likely correspond to the (Fe-Ni) phase, are approximately 10 to $20 \mathrm{~nm}$ in size and are spheroidal in shape. The larger grains, likely the Ag phase, are 50 to $150 \mathrm{~nm}$ in diameter and spherical in shape. A thin $(\sim 3 \mathrm{~nm})$ surface layer is visible on the smaller as-prepared powder grains. As evidenced by the XRD analyses of the as-prepared powder (Figure 1), the surface layer is likely an oxide (e.g., $\mathrm{NiFe}_{2} \mathrm{O}_{4}$ or $\mathrm{Fe}_{3} \mathrm{O}_{4}$,) that formed on the highly reactive nanoscale powders during preparation and handling. The heat treatment at $520^{\circ} \mathrm{C}$ for 2 hours in $\mathrm{H}_{2}$ effectively removes much of the surface oxide

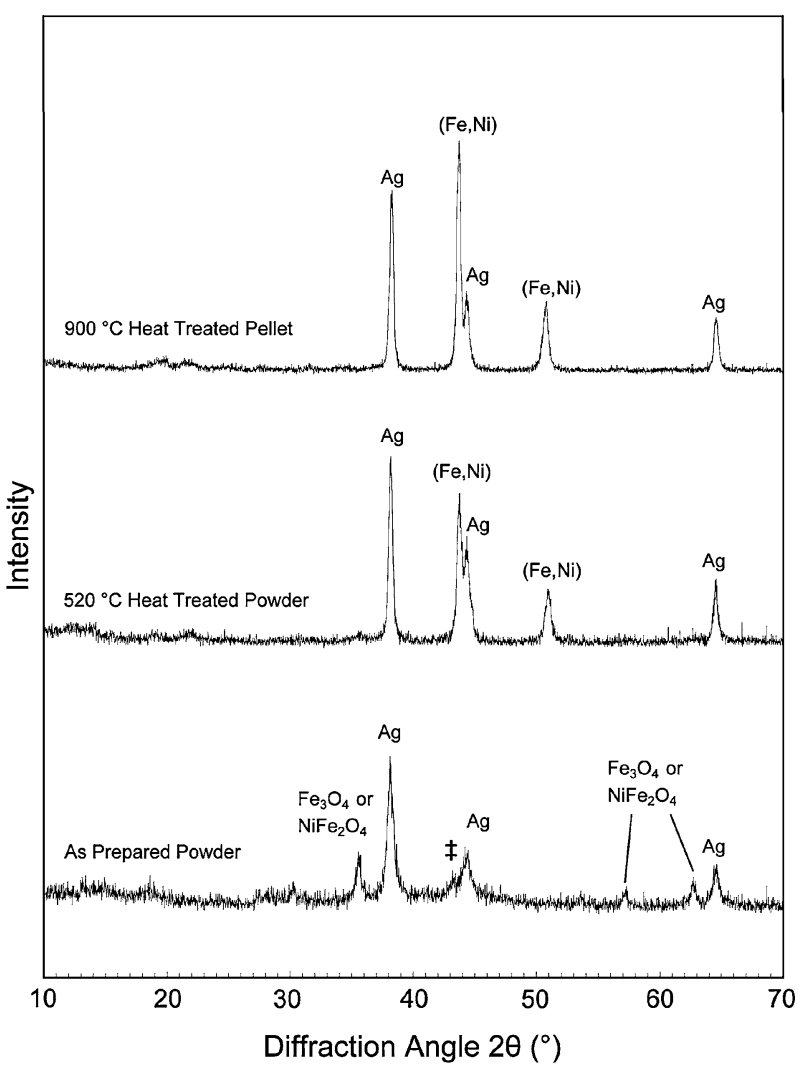

Fig. 1-XRD patterns of 30 Ag-70 Invar before heat treatment, after $520^{\circ} \mathrm{C}$ heat treatment in $\mathrm{H}_{2}$, and after sintering heat treatment at $900{ }^{\circ} \mathrm{C}$ in $\mathrm{H}_{2}$. The as-prepared powder has broad peaks indicating small grain size, multiple oxide peaks, and no clear evidence of the Invar phase. After heat treatment, the peaks become narrower as and more clearly defined as a result of grain growth, the oxide peaks disappear, and the $\gamma-(\mathrm{Fe}, \mathrm{Ni})$ Invar phase appears. Sintering and heat treating at $900{ }^{\circ} \mathrm{C}$ further narrows the peaks. The $\$$ peak may result from one or more of the following phases: $\mathrm{NiFe}_{2} \mathrm{O}_{3}, \mathrm{Fe}_{3} \mathrm{O}_{4}, \mathrm{Fe}, \mathrm{Ni}$, or $\gamma-(\mathrm{Fe}, \mathrm{Ni})$.

without dramatically increasing the grain size of the as-prepared $\gamma-(\mathrm{Fe}, \mathrm{Ni})$ powder (Figure 4). Grain growth is expected, however, in the Ag powder during the $520{ }^{\circ} \mathrm{C}$ heat treatment.

The SEM examination of polished cross sections shows that ternary Ag-Fe-Ni alloys that are compacted 


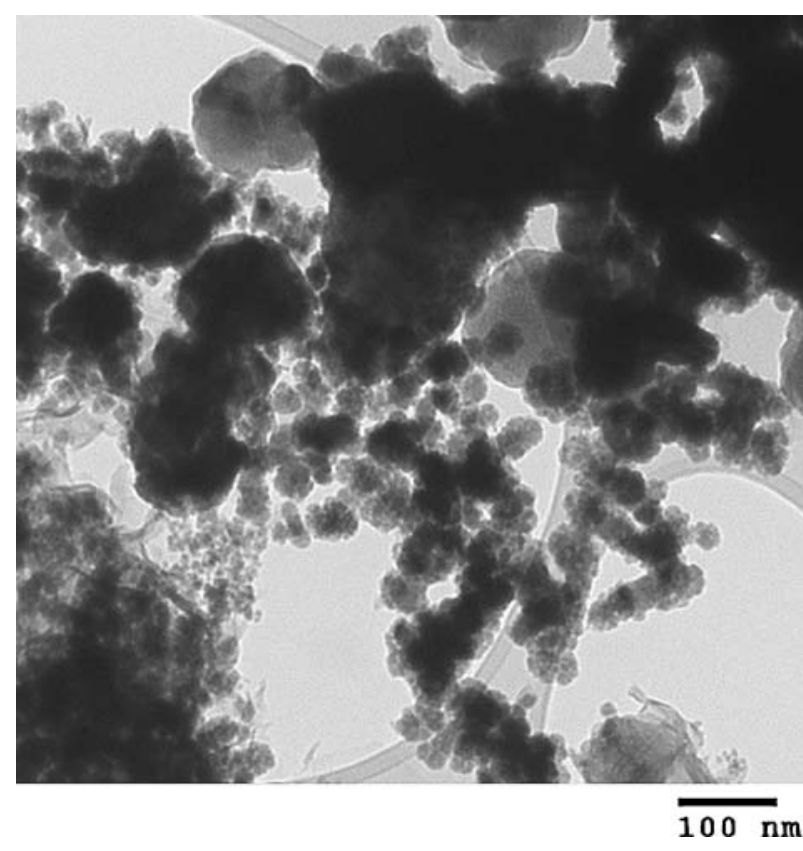

Fig. 2-TEM micrograph of as-prepared 50 Ag-50 Invar powder. The larger grains correspond to the $(\mathrm{Ag})$ phase and measure approximately 50 to $150 \mathrm{~nm}$ in diameter. The smaller grains are of $\mathrm{Fe}$ and $\mathrm{Ni}$ and measure approximately 10 to $20 \mathrm{~nm}$ in diameter.

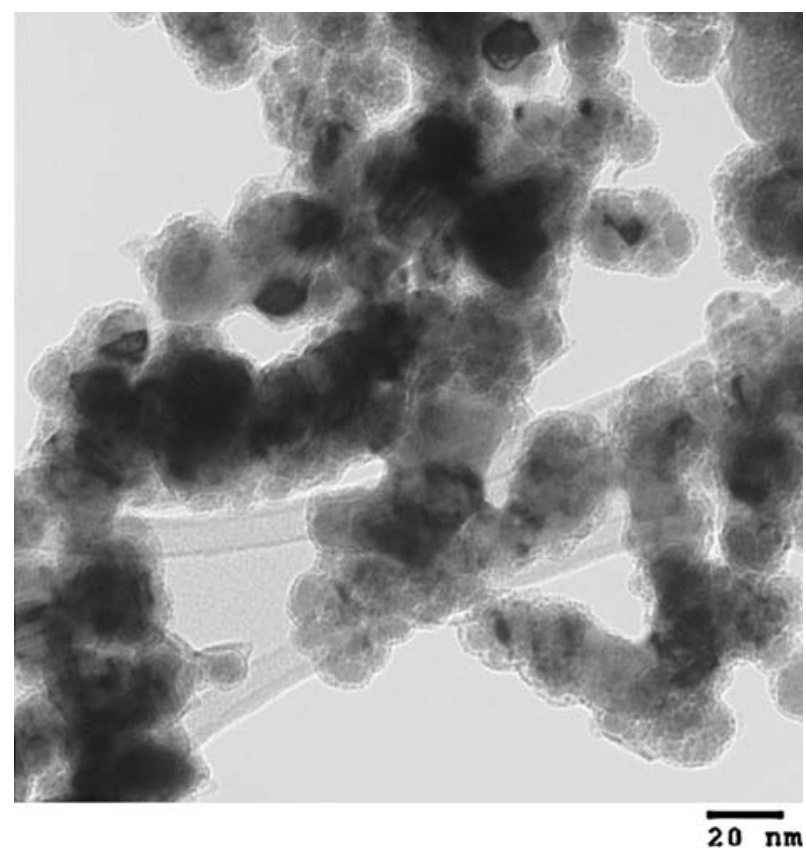

Fig. 3-TEM micrograph of as-prepared $50 \mathrm{Ag}-50$ Invar powder, showing a close view of the small $(\mathrm{Fe}, \mathrm{Ni})$ grains. The $(\mathrm{Fe}, \mathrm{Ni})$ grains have a thin layer $(\sim 3 \mathrm{~nm})$ of $\mathrm{Fe}$ or $\mathrm{Ni}$ oxides that was formed when the grains were exposed to air during synthesis.

and sintered at $900{ }^{\circ} \mathrm{C}$ for 1 hour in $\mathrm{H}_{2}$ consist of a uniform distribution of $\mathrm{Ag}$ and $\gamma-(\mathrm{Fe}, \mathrm{Ni})$ Invar grains (Figure 5). Samples of high Ag content have a continuous $(\mathrm{Ag})$ matrix with dispersed Invar, while Ag-Invar samples of high iron and nickel content show continuous

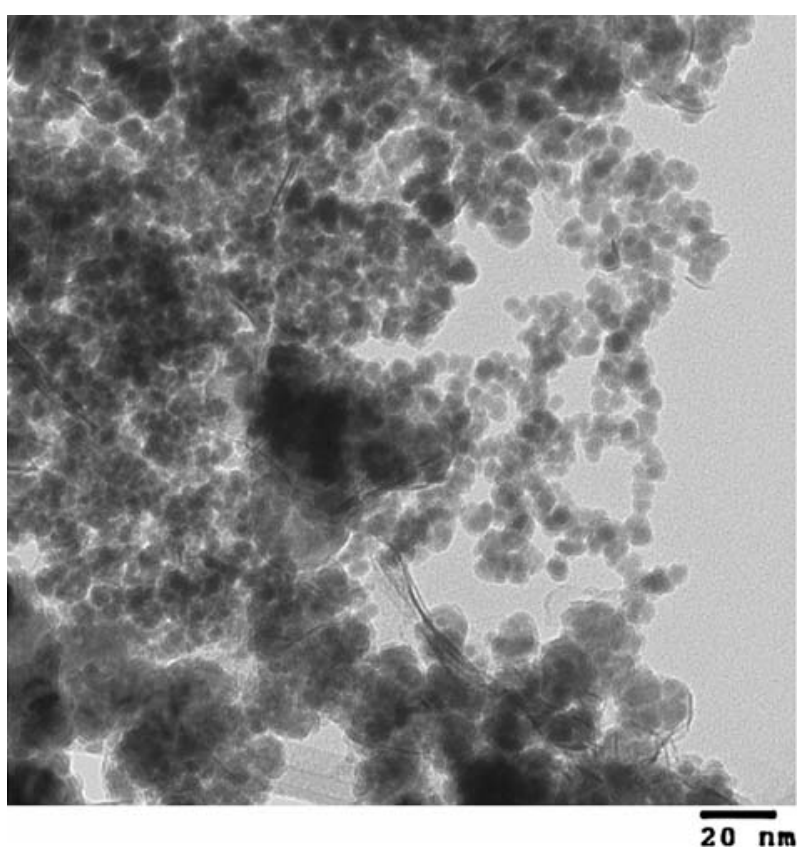

Fig. 4-TEM micrograph of $30 \mathrm{Ag}$-70 Invar powder after heat treating in $\mathrm{H}_{2}$ at $520^{\circ} \mathrm{C}$. The oxide layer has been reduced, leaving small $\gamma-(\mathrm{Fe}, \mathrm{Ni})$ and large $\mathrm{Ag}$ grains. The grains of $(\mathrm{Fe}, \mathrm{Ni})$ have not grown, though the $\mathrm{Ag}$ grains are expected to have grown.

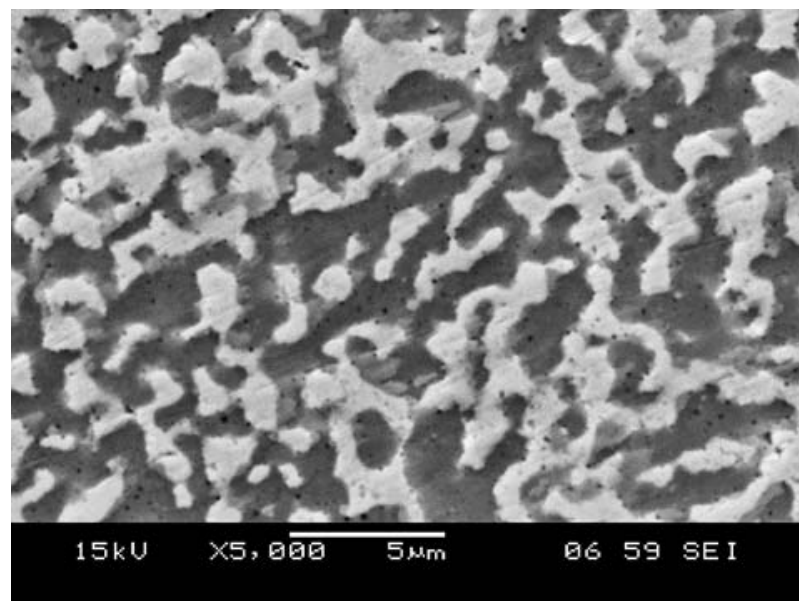

Fig. 5-SEM micrograph of sintered 50 Ag-50 Invar pellet. The lighter phase is $\mathrm{Ag}$, the darker phase is $\gamma-(\mathrm{Fe}, \mathrm{Ni})$ Invar, and the small black spots are pores. Diagonal scratches are artifacts of polishing with $0.05-\mu \mathrm{m} \mathrm{Al}_{2} \mathrm{O}_{3}$.

Invar with a dispersed silver phase. Samples of intermediate Ag content appear to have continuous Ag and continuous Invar phases. Average grain size for the $50 \mathrm{Ag}-50$ Invar sample after annealing at $900{ }^{\circ} \mathrm{C}$ for 1 hour is approximately $0.8 \mu \mathrm{m}$, and the grain morphology is equiaxed. As shown in Table II, the densities of sintered pellets are generally high ( $>90$ pct of theoretical for most samples), but some porosity is present in the sintered Ag-Invar alloys. Additional heat treatment time at $900{ }^{\circ} \mathrm{C}$ is expected to increase the densities of the sintered powder compacts. 
Table II. Sample Identification and Densities of the Sintered Ag-Fe-Ni Pellets

\begin{tabular}{lccc}
\hline $\begin{array}{l}\text { Nominal } \\
\text { Composition }\end{array}$ & $\begin{array}{c}\text { Experimental } \\
\text { Density } \\
\mathrm{g} / \mathrm{cm}^{3}\end{array}$ & $\begin{array}{c}\text { Theoretical } \\
\text { Density } \\
\mathrm{g} / \mathrm{cm}^{3}\end{array}$ & $\begin{array}{c}\text { Percent of } \\
\text { Theoretical } \\
\text { Density }\end{array}$ \\
\hline 90Ag-10Invar & 9.62 & 10.17 & 94.6 \\
80Ag-20Invar & 9.71 & 9.87 & 98.4 \\
70Ag-30Invar & 9.35 & 9.59 & 97.5 \\
60Ag-40Invar & 8.12 & 9.33 & 87.0 \\
50Ag-50Invar & 8.67 & 9.08 & 95.5 \\
40Ag-60Invar & 8.14 & 8.84 & 92.1 \\
30Ag-70Invar & 7.83 & 8.61 & 90.9 \\
20Ag-80Invar & 8.11 & 8.40 & 96.5 \\
10Ag-90Invar & 7.64 & 8.19 & 93.3 \\
\hline
\end{tabular}

\section{B. Thermal Expansion Behavior}

Average Ag-Invar values for a temperature range of $20{ }^{\circ} \mathrm{C}$ to $100{ }^{\circ} \mathrm{C}$, as determined by thermomechanical analysis, are provided in Table III and represented graphically in Figure 6. Also included in Figure 6 are CTE curves that were calculated using two different models for thermal expansion. The linear rule-of-mixtures model is a weighted average of the CTEs of Ag and Invar by their volume fraction, and is defined in Eq. [4] as

$$
\alpha=\alpha_{1} V_{1}+\alpha_{2} V_{2}
$$

where $V_{1}$ and $V_{2}$ are the volume fractions of silver and Invar, and $\alpha_{1}$ and $\alpha_{2}$ are the CTEs of the respective components. Differences in thermal expansion of the components in a composite or multiple-phase alloy system may, however, give rise to thermally induced stresses that can influence the thermal expansion behavior of a composite body. For this reason, the simple volumetric rule-of-mixtures model is not typically used to predict the CTE of composite or alloy systems. The most commonly used procedure for the prediction of the thermal expansion coefficient in two-phase alloys is the Turner formula, which

Table III. Theoretical and Experimental Coefficients of Thermal Expansion; Typical Property Values for Silver ${ }^{[24]}$ and Invar ${ }^{[17,25]}$ are Included for Comparison

\begin{tabular}{lccc}
\hline Nominal & $\begin{array}{c}\text { Rule-of- } \\
\text { Mixtures } \\
\text { CTE, } \\
\text { Composition }\end{array}$ & $\begin{array}{c}\text { Turner } \\
\text { Model } \\
\text { CTE, }\end{array}$ & $\begin{array}{c}\text { Experimental } \\
\text { CTE, }\end{array}$ \\
\hline 90Ag-10Invar & 17.6 & 15.8 & 17.6 \\
80Ag-20Invar & 15.4 & 12.8 & 13.0 \\
70Ag-30Invar & 13.4 & 10.5 & 11.6 \\
60Ag-40Invar & 11.5 & 8.54 & 10.3 \\
50Ag-50Invar & 9.72 & 6.97 & 8.76 \\
40Ag-60Invar & 8.01 & 5.66 & 7.42 \\
30Ag-70Invar & 6.40 & 4.54 & 5.23 \\
20Ag-80Invar & 4.86 & 3.58 & 3.82 \\
10Ag-90Invar & 3.40 & 2.74 & 3.37 \\
100 Ag (ref) & 19.6 & 19.6 & - \\
100 Invar (ref) & 2.01 & 2.01 & - \\
\hline
\end{tabular}

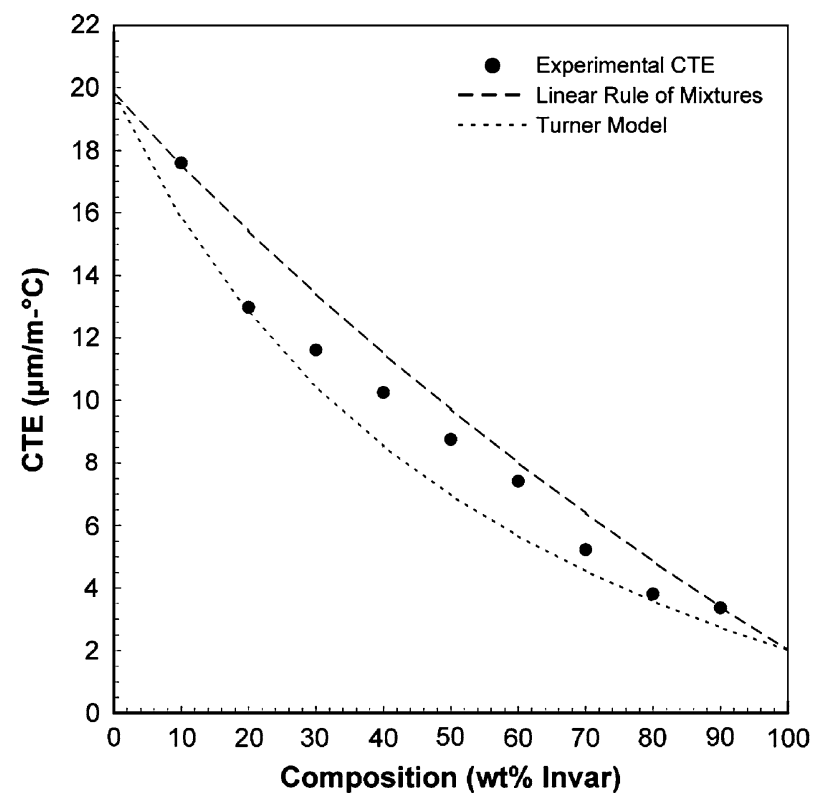

Fig. 6-Average coefficients of thermal expansion from $20^{\circ} \mathrm{C}$ to $100{ }^{\circ} \mathrm{C}$ for sintered Ag-Invar alloys. The data points correspond to experimental values, the dotted line corresponds to the prediction by Turner's formula, and the dashed line corresponds to the prediction by the volumetric rule of mixtures.

takes into account the ability of the stiffer phase to constrain the expansion of the more compliant phase. The Turner model gives the thermal expansion $\alpha$ of a composite as

$$
\alpha=\frac{\alpha_{1} E_{1} V_{1}+\alpha_{2} E_{2} V_{2}}{E_{1} V_{1}+E_{2} V_{2}}
$$

where $\alpha_{1}$ and $\alpha_{2}, V_{1}$ and $V_{2}$, and $E_{1}$ and $E_{2}$ are, respectively, the coefficients of thermal expansion, volume fractions, and elastic moduli of phases 1 and 2 in a composite. ${ }^{[22]}$ Equation [5] assumes only hydrostatic stresses in the two phases and uses the elastic modulus of each component to account for changes in the expansion due to thermally induced stresses.

The TMA data show a reduction in the average CTE with increasing Invar content. All of the average CTEs fall within the lower bound of the Turner model and the upper bound of the rule-of-mixtures model, indicating that the Fe:Ni ratios in the sintered alloys closely match the desired $64 \mathrm{Fe}-36 \mathrm{Ni}$ ratio for the Invar phase. The good fit between the measured and predicted values also indicates that the $\mathrm{Ag}$ and Invar phases in the sintered alloys are metallurgically distinct, i.e., that little Fe and $\mathrm{Ni}$ diffuses into the (Ag) phase and that little Ag diffuses into the $\gamma-(\mathrm{Fe}, \mathrm{Ni})$ Invar phase. This desirable phase separation was verified by EDS compositional dot mapping of several sintered samples.

Figure 7 illustrates the effect of increased $\mathrm{Ag}$ on the thermal expansion behavior of Ag-Invar alloys and compares the effect of temperature on the rate of thermal expansion. As noted previously, Invar and nearInvar alloys exhibit low thermal expansion near ambient temperatures due to a magnetovolume effect. Invar's low expansion behavior, however, becomes less 


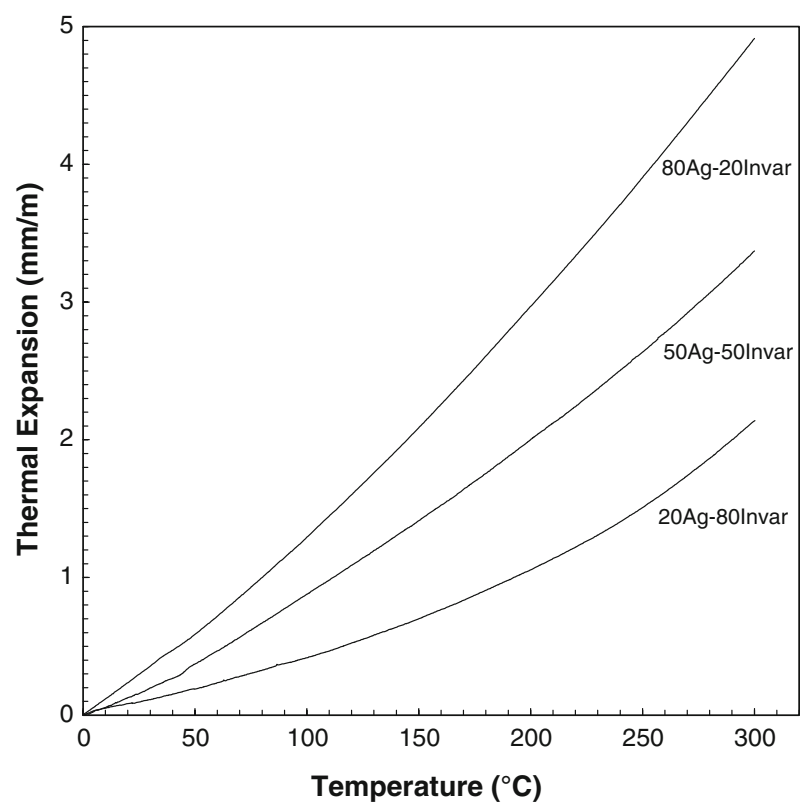

Fig. 7-Comparison of the thermal expansion behavior of 20Ag80Invar, 50Ag-50Invar, and $80 \mathrm{Ag}$-20Invar after sintering at $900^{\circ} \mathrm{C}$ for $1 \mathrm{~h}$.

pronounced at higher temperatures as the magnetic phase transformation goes to completion and the thermal expansion is no longer balanced by magnetostriction. As shown in Figure 7, the Invar effect is more apparent below temperatures of about $225^{\circ} \mathrm{C}$ and in samples of higher Invar content. For example, the 20 Ag-80 Invar sample has a low CTE at lower temperatures, but its thermal expansion increases dramatically at higher temperatures, as the Invar effect disappears. This behavior is also observed in samples of higher $\mathrm{Ag}$ content, although the changes in CTE for high Ag alloys at higher temperatures are not as pronounced as in alloys of high $\mathrm{Fe}-\mathrm{Ni}$ content.

\section{Conductivity of Ag-Invar Alloys}

The measured electrical conductivity and estimated thermal conductivity of sintered Ag-Invar alloys as a function of Invar content are shown in Figure 8 and Table IV. Thermal conductivities of Ag-Invar alloys are calculated using the Wiedemann-Franz law (Eq. [3]). Since thermal conductivities are linearly related to electrical conductivities, the results are simply a scaled version of the electrical conductivity results, with thermal conductivity decreasing with Invar content.

Figure 8 includes several curves that represent predicted conductivity values based on several different models for conductivity of two-phase composites or mixtures. Two simple models - conductors in parallel and conductors in series - represent the expected upper and lower bounds of the Ag-Invar alloy conductivities. ${ }^{[26]}$ The parallel model represents the Ag and Invar phases as plates oriented parallel to the heat or current flow, and composite conductivity $k_{\text {mix }}$ in this model is given as

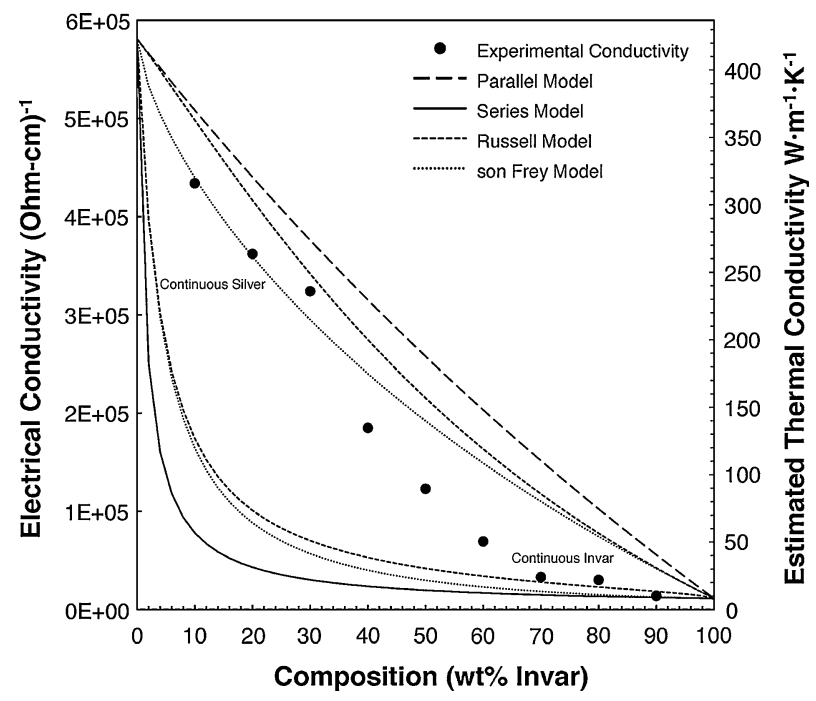

Fig. 8-Electrical conductivity and estimated thermal conductivity of sintered Ag-Invar alloys compared with various model predictions. ${ }^{[26]}$ The values predicted by the Russell and son Frey models for two-phase mixtures closely match the experimental values for high Ag samples (continuous silver with dispersed Invar) and low Ag samples (continuous Invar with dispersed silver).

$$
k_{\text {mix }}=V_{1} k_{1}+V_{2} k_{2}
$$

where $k_{1}$ and $k_{2}$ represent the conductivities of the silver and Invar phases and $V_{1}$ and $V_{2}$ are the volume fraction of the respective phases. Under these conditions, the electrical and heat conduction are dominated by the better conductor. The series model represents the Ag and Invar phases as plates oriented perpendicular to the heat or current flow; composite conductivity $k_{\text {mix }}$ in this model is defined as

$$
k_{\text {mix }}=\frac{1}{\frac{V_{1}}{k_{1}}+\frac{V_{2}}{k_{2}}}
$$

In the series model, the electrical and heat conduction are dominated by the poorer conductor.

The Russell and son Frey models for two-phase mixtures predict composite conductivity values that lie between those of the parallel and series models. ${ }^{[26]}$ Both the Russell and son Frey models assume a discontinuous phase with volume fraction $V_{d}$ and conductivity $k_{d}$ dispersed within a continuous phase with conductivity $k_{c}$, and both models are based on cubes dispersed in a cubic array geometry. The Russell model assumes linear isotherms and predicts the composite conductivity $k_{\text {mix }}$ as

$$
k_{\text {mix }}=k_{c}\left[\frac{1-V_{d}^{2 / 3}+\frac{k_{d}}{k_{c}} V_{d}^{2 / 3}}{1-V_{d}^{2 / 3}+V_{d}+\frac{k_{d}}{k_{c}}\left(V_{d}^{2 / 3}-V_{d}\right)}\right]
$$

while the son Frey model assumes linear heat flow and predicts the composite conductivity $k_{\text {mix }}$ as

$$
k_{\operatorname{mix}}=k_{c}\left[\frac{1-V_{d}^{1 / 3}+V_{d}+\frac{k_{c}}{k_{d}}\left(V_{d}^{1 / 3}-V_{d}\right)}{1-V_{d}^{1 / 3}+\frac{k_{c}}{k_{d}} V_{d}^{1 / 3}}\right]
$$


Table IV. Measured Experimental Electrical Conductivities of Sintered Ag-Invar Pellets, and Estimated Thermal Conductivities for the Ag-Invar Alloys Based on the Wiedemann-Franz Law; Parallel and Series Model Predicted Values, as well as Typical Property Values for Silver ${ }^{[24]}$ and Invar, ${ }^{[17]}$ are Included for Comparison

\begin{tabular}{|c|c|c|c|c|}
\hline \multirow[b]{2}{*}{ Nominal Composition } & \multicolumn{3}{|c|}{ Electrical Conductivity, $\mathrm{Ohm}^{-1} \cdot \mathrm{cm}^{-1}$} & \multirow{2}{*}{$\begin{array}{c}\text { Thermal Conductivity, } \mathrm{W} \cdot \mathrm{m}^{-1} \cdot \mathrm{K}^{-1} \\
\text { Experimental (Estimated) }\end{array}$} \\
\hline & Parallel Model & Serial Model & Experimental & \\
\hline 90Ag-10Invar & $5.1 \times 10^{5}$ & $7.8 \times 10^{4}$ & $4.3 \times 10^{5}$ & 320 \\
\hline 80Ag-20Invar & $4.4 \times 10^{5}$ & $4.3 \times 10^{4}$ & $3.6 \times 10^{5}$ & 260 \\
\hline 70Ag-30Invar & $3.8 \times 10^{5}$ & $3.0 \times 10^{4}$ & $3.2 \times 10^{5}$ & 240 \\
\hline $60 \mathrm{Ag}$-40Invar & $3.2 \times 10^{5}$ & $2.4 \times 10^{4}$ & $1.9 \times 10^{5}$ & 140 \\
\hline 50Ag-50Invar & $2.6 \times 10^{5}$ & $2.0 \times 10^{4}$ & $1.2 \times 10^{5}$ & 90 \\
\hline $40 \mathrm{Ag}-60$ Invar & $2.0 \times 10^{5}$ & $1.7 \times 10^{4}$ & $6.9 \times 10^{4}$ & 50 \\
\hline 30Ag-70Invar & $1.5 \times 10^{5}$ & $1.5 \times 10^{4}$ & $3.3 \times 10^{4}$ & 24 \\
\hline $20 \mathrm{Ag}-80$ Invar & $1.0 \times 10^{5}$ & $1.3 \times 10^{4}$ & $3.0 \times 10^{4}$ & 22 \\
\hline 10Ag-90Invar & $5.6 \times 10^{4}$ & $1.2 \times 10^{4}$ & $1.4 \times 10^{4}$ & 41 \\
\hline $100 \mathrm{Ag}$ (ref) & $5.8 \times 10^{5}$ & - & - & 423 \\
\hline 100 Invar (ref) & $1.2 \times 10^{4}$ & - & - & 9 \\
\hline
\end{tabular}

Since the Russell and son Frey models are intended for composites with a minor phase dispersed within a major phase, the values predicted by the models are expected to be more accurate for Ag-Invar compositions closer to pure silver or pure Invar.

The experimental electrical conductivity values and calculated thermal conductivity values for Ag-Invar alloys match closely with the son Frey model for high Ag and low Ag content. The trends in conductivity may be explained through an examination of phase continuity through the microstructure. In samples of high $\mathrm{Ag}$ content, the (Ag) phase forms a continuous, highconductivity matrix that enables excellent electronic conduction through the sample; and the Invar phase in high Ag samples forms isolated, low-conductivity grains that do not significantly disrupt the flow of electrons. Similarly, at low Ag contents, the low-conductivity Invar phase is continuous, and the high-conductivity (Ag) exists only in isolated grains. In samples of low $\mathrm{Ag}$ composition, the conducting electrons are forced to move through the high resistivity Invar regions. Since the conductivity difference between pure $\operatorname{Ag}(\sigma=$ $\left.5.81 \times 10^{5} \mathrm{Ohm}^{-1} \cdot \mathrm{cm}^{-1}\right)$ and Invar $\left(\sigma=1.20 \times 10^{4}\right.$ $\left.\mathrm{Ohm}^{-1} \cdot \mathrm{cm}^{-1}\right)$ is so great, the loss of continuity in the (Ag) phase quickly drives the alloy conductivity to lower values. Conductivities for samples of intermediate $\mathrm{Ag}$ content are expected to transition along an S-shaped curve between the predicted values for continuous (Ag) and continuous Invar.

Differences between the predicted conductivities and the measured values may be due to the presence of pores, which serve as electron scattering centers and severely limit conduction. Since porosity has a thermal conductivity that is close to zero, the effective thermal conductivity $k_{\mathrm{eff}}$ of bulk materials with isolated pores may be estimated as

$$
k_{\mathrm{eff}}=k(1-\omega)
$$

where $k$ is the conductivity of the nonporous material and $\omega$ is the pore fraction. ${ }^{[26]}$ The effect of porosity is observed in the 60Ag-40Invar sintered pellet, which exhibits a relatively high porosity and a correspondingly low conductivity. Conductivity values may also be reduced due to the relatively high grain boundary to volume ratio in the fine-grained alloys, or to a small amount of dissolved $\mathrm{Fe}$ or $\mathrm{Ni}$ in the (Ag) phase. For highly conductive metals such as silver, even trace amounts of dissolved impurities will significantly decrease the alloy conductivity.

\section{Solubility and Phase Stability of Ag-Invar}

As noted in Section I-B, little published information on the phase development and ambient-temperature phase stability in the Ag-Fe-Ni ternary system is available. As such, the phase stability, microstructural development, and properties of ternary Ag-Fe-Ni alloys are still in need of thorough research.

This investigation has demonstrated that thermodynamically stable, two-phase Ag-Invar alloys are formed along a constant $64 \mathrm{Fe}-36 \mathrm{Ni}$ compositional line in the ternary system. The formation of two-phase Ag-Invar alloys is not entirely surprising, given the solubility information available in the Ag-Fe, Ag-Ni, and Fe-Ni binary phase diagrams. ${ }^{[23]}$ Solubility limits of $\mathrm{Fe}$ and $\mathrm{Ni}$ in the (Ag) solid solution at silver's melting temperature $\left(961.93^{\circ} \mathrm{C}\right)$ are 0.00337 and $0.7 \mathrm{wt}$ pct, respectively. These low solubility limits, even at high temperatures, indicate that $\mathrm{Fe}$ and $\mathrm{Ni}$ are unlikely to diffuse into (Ag) and thus unlikely to negatively affect electrical conductivity. Similarly, silver has low miscibility with iron and nickel, with limits of 0.0004 and 1.8 wt pet for $\mathrm{Ag}$ in $(\mathrm{Fe})$ and (Ni), respectively. The low solubility of $\mathrm{Ag}$ in iron and nickel, and vice versa, indicates that the critical ratio of $\mathrm{Fe}$ to $\mathrm{Ni}$ in the $\gamma-(\mathrm{Fe}, \mathrm{Ni})$ Invar phase should be maintained in the Ag-Invar alloys.

Since the Ag-Ni solubility limits are higher than those in $\mathrm{Ag}-\mathrm{Fe}$, the solid-state diffusion of $\mathrm{Ni}$ in the ternary Ag-Invar alloys represents a potential problem. Although EDS compositional dot mapping indicates that the desired phase separation is occurring, it is possible that some $\mathrm{Ni}$ may be dissolved in the (Ag) phase. Dissolved $\mathrm{Ni}$ atoms in the silver phase would simultaneously decrease the (Ag) conductivity and increase the Invar thermal expansion. Since solubility 
limits of $\mathrm{Ni}$ in $(\mathrm{Ag})$ and $\mathrm{Ag}$ in $(\mathrm{Ni})$ decrease with temperature, however, further atomic segregation and improved conductivity and CTEs may be achieved through aging heat treatments. It is expected that most dissolved Ni may be driven out of the $(\mathrm{Ag})$ phase by heating the Ag-Invar alloys to temperatures around 0.5 $T_{M}$ and holding for some time.

\section{CONCLUSIONS}

Nanoscale Ag-Fe-Ni ternary metal powders were prepared using a simple, ambient-temperature, solution-based chemical technique involving sodium borohydride reduction of metal nitrates. The Ag and Invar phases form upon annealing of the nanocrystalline Ag$\mathrm{Fe}-\mathrm{Ni}$ powder at $520{ }^{\circ} \mathrm{C}$ in $\mathrm{H}_{2}$, and sintering of the annealed powder in $\mathrm{H}_{2}$ at $900{ }^{\circ} \mathrm{C}$ leads to the formation of fine-grained, two-phase Ag-Invar alloys. Compositional dot mapping indicates nearly complete compositional separation between the $\mathrm{Ag}$ and $\gamma-(\mathrm{Fe}, \mathrm{Ni})$ Invar phases, resulting in high conductivity in the $\mathrm{Ag}$ and low thermal expansion in the Invar. The CTEs and electrical conductivities of the Ag-Invar alloys decrease with increasing Invar content, and specific values of CTE and conductivity are easily obtainable by this synthesis and processing approach. The CTE values closely match those predicted by common models, and the electrical conductivity values are described by simple models for two-phase mixtures. The thermal and electrical properties of the Ag-Invar alloys prepared by this method are comparable to some of the existing low CTE-high conductivity materials, indicating that a range of Ag-Invar alloys may be viable for use in thermal management applications. The simple synthesis, relatively low processing costs, small grain size, and isotropic properties of the ternary alloys may offer distinct advantages over some of the materials currently in use.

\section{ACKNOWLEDGMENTS}

The authors thank Clara Cho and Alex Epstein for their contributions to this project and the Franklin
W. Olin College of Engineering for partial support of the project through the Olin College Faculty Research Program.

\section{REFERENCES}

1. F.J. Dance and J.L. Wallace: Circuit World, 1982, vol. 9, pp. 4-7.

2. H. Baudry and G. Kersuzan: 5th Euro. Hybrid Microelectronics Conf. Proc., 1985, pp. 454-64.

3. W.R. Johannes and W. Johnson: Int. J. Microcircuits Elec. Pkg., 1994, vol. 17, pp. 135-42.

4. D.S. Copeland, M.K. Rahim, J.C. Suhling, G. Tian, P. Lall, and R.C. Jaeger: Proc. 2005 ASME Int. Mech. Engr. Congress Expo., 2005, pp. 185-98.

5. R.A. Koch, R. Watts, T. Benson-Tolle, and K. Strong: Proc. 48th Int. SAMPE Symp., 2003, pp. 84-89.

6. V. Sundaram, R. Tummala, B. Wiedenman, F. Liu, P.M. Raj, I.R. Abothu, S. Bhattacharya, M. Varadarajan, E. Bongio, and W. Sherwood: Proc. 56th Elec. Comp. Tech. Conf., 2006, pp. 1357-80.

7. C. Zweben: J. Adv. Mater., 2007, vol. 39 (1), pp. 3-10.

8. A. Luedtke: Adv. Eng. Mater., 2004, vol. 6 (3), pp. 142-44.

9. F. Spexarth, J. Cronin, and W. Johnson: Int. J. Microcircuits Elec. Pkg., 1995, vol. 18 (2), pp. 138-45.

10. F. Spexarth, J. Cronin, and W. Johnson: Adv. Pkg., 1995, Mar.Apr., pp. 36-38.

11. P. Yih and D.D.L. Chung: J. Mater. Sci., 1997, vol. 32 (11), pp. $2873-82$.

12. S. Jha: Proc. 45th Elec. Component Technol. Conf., 1995, pp. $542-$ 47.

13. D.E. Jech and J.L. Sepulveda: Int. Symp. Microelectron., SPIE Proc. 3235, 1997, pp. 90-96.

14. J. Stolk and A. Manthiram: Metall. Mater. Trans. A, 2000, vol. 31A, pp. 2396-98.

15. R.M. Bozorth: Ferromagnetism, Van Nostrand, Princeton, NJ, 1978, p. 641

16. D.F. Berry: ASM Handbooks Online, ASM INTERNATIONAL, Materials Park, OH, 2002, vol. 2.

17. E.L. Frantz: ASM Handbooks Online, ASM INTERNATIONAL, Materials Park, OH, 2002, vol. 2.

18. W. Johnson and S. Nelson: Proc. IEPS Conf., 1995, pp. 228-35.

19. J. Stolk, M. Gross, D. Stolk, and A. Manthiram: J. Mater. Res., 2001, vol. 16 (2), pp. 340-43.

20. L.J. van der Pauw: Philips Technol. Rev., 1958, vol. 20, pp. 220-24.

21. F.J. Blatt: Physics of Electronic Conduction in Solids, McGraw-Hill Book Company, New York, NY, 1968.

22. A.A. Fahmy and A.N. Ragai: J. Appl. Phys., 1970, vol. 41, pp. $5108-11$

23. ASM Handbooks Online, ASM INTERNATIONAL, Materials Park, OH, 2002, vol. 3.

24. ASTM B 742-90(2006), ASTM, Philadelphia, PA, 2006.

25. ASTM F 1684-06, ASTM, Philadelphia, PA, 2006.

26. D.R. Poirier and G.H. Geiger: Transport Phenomena in Materials Processing, TMS, Warrendale, PA, 1998, pp. 208-11. 